\title{
ODOVTOS
}

International Journal of Dental Sciences

https://revistas.ucr.ac.cr/index. php/Odontos I ISSN: 2215-3411

CASE REPORT

DOI: $10.15517 /$ IJDS.2021.44324

Recibido:

8-VIII-2020

Aceptado:

29-IX-2020

Publicado en línea: $22-X-2020$
Abordaje transcervical en el síndrome de Eagle.

Reporte de caso clínico

\section{Transcervical Approach in Eagle Syndrome. Clinical Case Report}

José E. Miranda Villasana DDS, MSc; Diego A. Ayala González DDS²;

Josué Gallardo Caudillo DDS, MSc ${ }^{3}$; José E. Palma Medina DDS ${ }^{4}$

1. Jefe del Servicio de Cirugía Oral y Maxilofacial del Hospital Regional "General Ignacio Zaragoza", ISSSTE-UNAM, Ciudad de México, México.

2. Residente de tercer año de Cirugía Oral y Maxilofacial del Hospital Regional "General Ignacio

Zaragoza", ISSSTE-UNAM, Ciudad de México, México.

3. Cirujano oral y maxilofacial del Servicio de Cirugía Oral y Maxilofacial del Hospital Regional

"General Ignacio Zaragoza", ISSSTE-UNAM, Ciudad de México, México.

4. Residente de primer año de Cirugía Oral y Maxilofacial del Hospital Regional "General Ignacio

Zaragoza", ISSSTE-UNAM, Ciudad de México, México.

Autor para correspondencia: Dr. Diego A. Ayala González - diego.ago@outlook.com

RESUMEN: El síndrome de Eagle es una enfermedad rara responsable de múltiples síntomas de cabeza y cuello, resultado de un alargamiento del proceso estiloideo u osificación del ligamento estilohioideo comprimiendo estructuras neurovasculares adyacentes, hay dos variantes, el clásico caracterizado principalmente por dolor y disfagia y la variante carotídea distinguido con dolor y en ocasiones isquemia cerebral. Describimos un reporte de caso clínico de un paciente femenino de 45 años, quien experimentaba dolor cervical de lado izquierdo, realizando el protocolo completo de dolor miofascial del Hospital Regional "General Ignacio Zaragoza" ISSSTE de la Ciudad de México, el estudio de tomografía computada evidenció una elongación de $50 \mathrm{~mm}$ del proceso estiloideo, confirmando el diagnóstico, enfocando el artículo en la descripción anatómico-quirúrgica.

PALABRAS CLAVE: Compartimento post-estiloideo; Compartimento pre-estiloideo; Dolor miofascial; Osificación; Ligamento; Proceso estiloideo (fuente: MeSH NLM). 
ABSTRACT: Eagle syndrome is a rare disease responsible for multiple head and neck symptoms, resulting from an elongation of the styloid process or ossification of the stylohyoid ligament compressing adjacent neurovascular structures. There are two variants, the classic one characterized mainly by pain and dysphagia and the carotid variant distinguished with pain and sometimes cerebral ischemia.

We describe a clinical case report of a 45-year-old female patient, who experienced left cervical pain, performing the complete myofascial pain protocol of the Regional Hospital "General Ignacio Zaragoza" ISSSTE in Mexico City, resulting in a $50 \mathrm{~mm}$ elongation of the styloid process in the CT scan, confirming the diagnosis, and focusing the article on the anatomical-surgical description.

KEYWORDS: Pre-styloid compartment; Post-styloid compartment; Myofascial pain; Ossification; Ligament; Styloid process (source: MeSH NLM).

\section{INTRODUCCIÓN}

El síndrome de Eagle es una enfermedad rara, responsable de síntomas polimórficos de cabeza y cuello, resultado de un alargamiento del proceso estiloideo u osificación del ligamento estilohioideo comprimiendo estructuras neurovasculares adyacentes $(1,2)$.

De acuerdo con Eagle, el proceso estiloideo se encuentra elongado en un 4\% de la población, pero solo el $0,0016 \%$ es sintomático (1).

Las mujeres son mayormente afectadas en comparación con los hombres (3:1), y la edad media se encuentra entre la cuarta y sexta década de la vida.

Los síntomas más comunes reportados son dolor cervical exacerbado por la hiperextensión 0 movimientos rápidos del cuello, dolor de cabeza, sensación de cuerpo extraño en la faringe, disfagia, odinofagia, mareo o en ocasiones disfonía 0 trismus $(2,3)$. Rara vez puede ocasionar síndromes neuropáticos por una obstrucción vascular. La examinación clínica revela dolor a la palpación del proceso estiloideo a través de la fosa tonsilar $(1,2)$.
El diagnóstico del síndrome de Eagle está basado en la combinación de signos clínicos sugestivos, dolor a la palpación del proceso estiloideo en la región tonsilar, y una examinación tomográfica de la elongación del proceso $(2,3)$.

Varias teorías han sido propuestas para la explicación de la elongación y el cambio de angulación del proceso estiloideo: osificación del ligamento estilohioideo por cuestiones traumáticas, elongación congénita bajo la persistencia de cartílago vestigial, fractura directa del proceso estiloideo ocasionando una formación anormal elongada por la mal unión (2).

Múltiples tratamientos han sido sugeridos por la literatura: tratamiento médico y quirúrgico. El tratamiento curativo propuesto es la estiloidectomía transcervical, el cual permite una resolución completa de la mayoría de los síntomas.

El propósito de este reporte es presentar un paciente con diagnóstico clínico y tomográfico de síndrome de Eagle tratado quirúrgicamente por medio de un abordaje transcervical, en el Hospital Regional "General Ignacio Zaragoza" ISSSTE en la Ciudad de México, realizando el protocolo inicial 
de dolor miofascial y valorando las implicaciones anatómico-quirúrgicas.

\section{REPORTE DE CASO}

Paciente femenino de 45 años quien acude a consulta externa de cirugía oral y maxilofacial del Hospital Regional "General Ignacio Zaragoza" ISSSTE en la Ciudad de México, con un motivo de consulta de un historial de dolor cervical de lado izquierdo acentuado a la rotación ipsilateral y flexión cervical, con una mínima sensación de objeto extraño en la faringe, el cual es sometido a protocolo completo de dolor miofascial, realizando historia clínica completa, sin antecedentes sistémicos 0 alergias a medicamentos, estudio de laboratorio completo dentro de parámetros normales, a la examinación tomográfica se observa elongación del proceso estiloideo izquierdo, se realiza medición con la regla automática del programa, con una longitud aproximada de $50 \mathrm{~mm}$. Se comenta con la paciente el diagnóstico clínico y tomográfico (Figura 1. A, Figura 1. B) aceptando las condiciones de realizar extracción quirúrgica del proceso estiloideo elongado por medio de abordaje transcervical, previo consentimiento informado. En la sala de operaciones, bajo anestesia general balanceada con intubación orotraqueal, previa asepsia y antisepsia y colocación de campos estériles, se infiltra lidocaína 2\% con epinefrina 1: 100000 para fines hemostáticos, con el cuello en hiperextensión y rotación lateral hacia el lado derecho, se comienza con el marcaje de la zona a intervenir (Figura 2. A), se efectúa la incisión inicial de $405 \mathrm{~cm}$ en longitud en el ángulo de la mandíbula siguiendo anterocaudalmente el trayecto del músculo esternocleidomastoideo utilizando bisturí con hoja \#15, se incide piel y tejido celular subcutáneo (Figura 2. B) y se continua con electrobisturí incidiendo la fascia cervical superficial y el músculo platisma, los extremos de la incisión se disecan para permitir la retracción de la piel en la parte anterior y posterior, se prosigue con la disección de la fascia cervical profunda y se alcanza el borde anterior del músculo esternocleidomastoideo, mediante una tracción posterolateral del mismo músculo, tomando en cuenta el trayecto y el daño potencial a la rama mandibular marginal del nervio facial y del nervio auricular mayor, rama del plexo cervical, subsecuentemente el espacio comprendido entre la glándula parótida y el vientre posterior del músculo digástrico es explorado mediante disección digital hasta alcanzar el ápex del proceso estiloideo (Figura 2. C), la separación de la apófisis estiloides es completada con disección subperióstica del proceso, y con unas pinzas Rochester se concluye con la fractura y la resección del espécimen (Figura 2. D, Figura 2. E). Se verifica hemostasia y se termina el procedimiento con el cierre subcutáneo mediante sutura de ácido poliglicólico 3-0 y cierre de piel con nylon 6-0.

La emersión anestésica se realiza por lisis metabólica, se aspiran secreciones nasales y orales, y se extuba la paciente al cumplir criterios. Se traslada al área de recuperación, consciente, despierta. Durante su recuperación no se presentó incidente alguno, con un tiempo quirúrgico de 30 minutos, un sangrado intraoperatorio de $15 \mathrm{~mL}$.

Al día siguiente, se realiza la valoración motora del nervio mandibular marginal y sensitiva del nervio auricular mayor sin presentar daño alguno, sin datos de formación de seromas y/0 hematomas, se decide egreso hospitalario con esquema de antibiótico y analgésico, sin complicaciones ni eventualidades. Se cita a los 8 días por medio de consulta externa para valoración de la herida, con adecuado proceso de cicatrización, indicando masoterapia y termoterapia. 

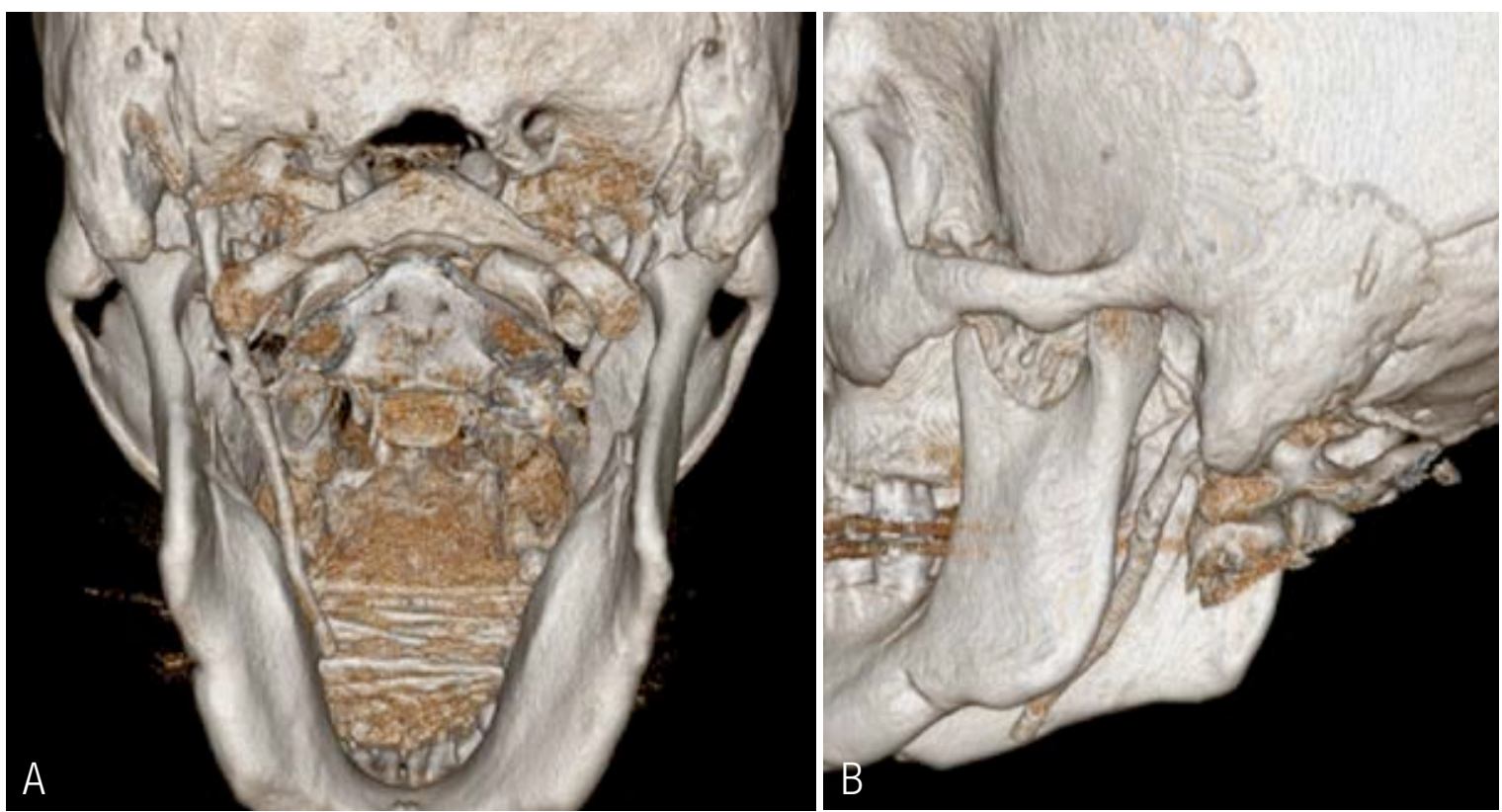

Figura 1. Estudio de tomografía computarizada. A. Estudio tomográfico con reconstrucción en 3D, vista posterior, observándose la elongación del proceso estiloideo de lado izquierdo. B. Estudio tomográfico con reconstrucción en 3D, vista lateral de lado izquierdo, observándose la elongación y su extensión del proceso estiloideo.

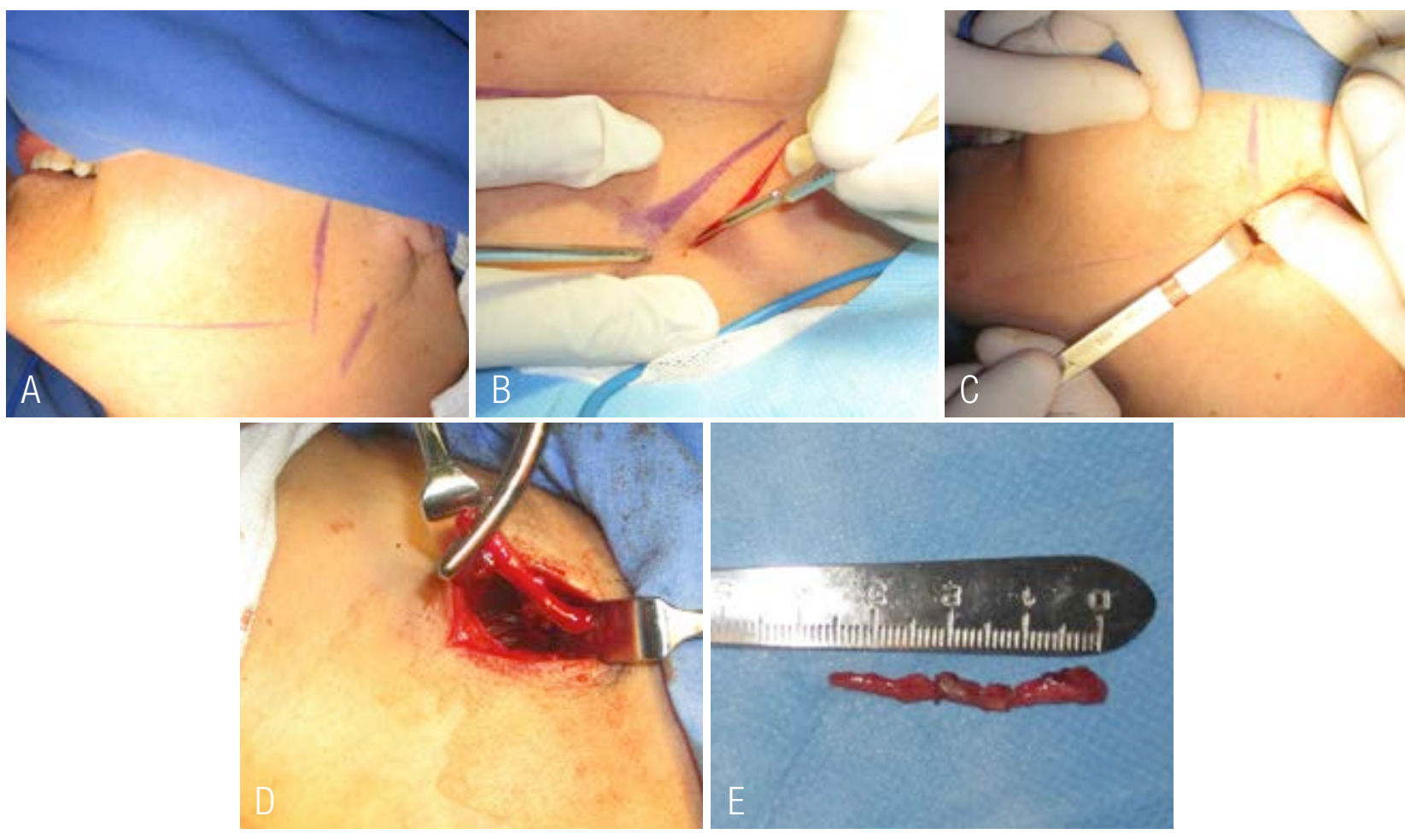

Figura 2. Fotografías intraoperatorias. A. Marcaje quirúrgico del borde posterior e inferior mandibular y borde anterior del músculo esternocleidomastoideo. B. Incisión realizada en piel y tejido celular subcutáneo con hoja de bisturí \#15. C. Disección roma digital para localizacion del ápice del proceso estiloideo. D. Fractura y extracción con pinzas Rochester del proceso estiloideo. E. Espécimen y medición del proceso estiloideo de alrededor de $3.5 \mathrm{~cm}$ de longitud. 


\section{DISCUSIÓN}

El complejo estilohioideo se deriva embriológicamente del segundo arco branquial a través del cartílago de Reichert, conformando: el estapedio, proceso estiloideo, ligamento estilohioideo, la asta menor y la parte superior del cuerpo del hueso hioides (Figura 3) $(2,4)$.

El proceso estiloideo emerge de la porción inferior del peñasco del hueso temporal, y se dirige inferior y oblicuo, anterior y medio a la fosa tonsilar (2). El proceso estiloideo está situado entre la rama de la mandíbula y las vértebras cervicales, constituyendo un sitio de inserción de múltiples músculos y ligamentos, denominados como diafragma estiloideo. La dirección, longitud y la relación con las estructuras neurovasculares del proceso estiloideo varía enormemente en cada individuo.

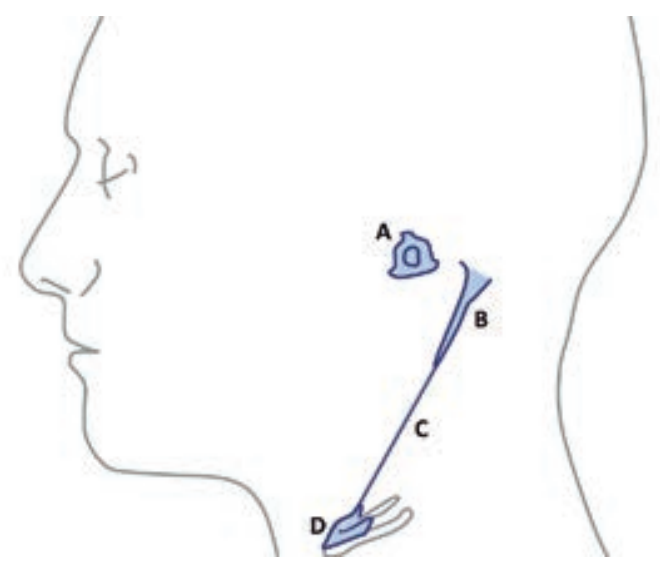

Figura 3. Esquema representativo del segundo arco faríngeo. A. Estapedio; B. Proceso estiloideo; C. Ligamento estilohioideo y D. Parte superior del cuerpo y asta menor del hueso hioides.

El diafragma estiloideo es una estructura músculo-fascial que se extiende desde la base de cráneo superiormente hacia el hueso hioides inferiormente, el músculo esternocleidomastoideo y la fascia cervical superficial lateralmente, el músculo constrictor superior de la faringe y la fascia peri faríngea medialmente. El proceso estiloideo separa el espacio faríngeo lateral en dos espacios: pre estiloideo y retro estiloideo $(3,5)$.
Anormalidades del complejo estilohioideo fueron primeramente descritas en animales por Vesalius en 1543. La primera descripción en humanos fue publicada por Marchetti en 1656 (6). El síndrome de Eagle, fue descrito por primera vez por Watt Weems Eagle en 1937, denominado como "estilalgia" es una elongación sintomática del proceso estiloideo y/o calcificación del ligamento estilohioideo (7). Un proceso estiloideo elongado está definido con una longitud de al menos de 25 $\mathrm{mm}$, se ha reportado que solo del $4-10 \%$ de los pacientes con el proceso elongado reportan dolor (8). La frecuencia del síndrome está estimada de 4-8 por 10000 en la población (9).

Eagle fue el primero en describir dos síndromes asociados: 1) síndrome de Eagle clásico fue descrito como dolor, disfagia, y la sensación de cuerpo extraño que se presenta inmediatamente después de una tonsilectomía y, 2) variante congénita, también descrita como síndrome estilohioideo 0 estilo carotídeo caracterizado con dolor en la región parietal/peri orbitaria, disturbios visuales, signos y síntomas de compresión carotídea (pre-síncope, síncope o eventos isquémicos transitorios) causado por una osificación del ligamento estilohioideo $(7,10,11)$.

La etiología del dolor asociado con el síndrome de Eagle puede ser multifactorial, pero en la mayoría de los casos es el resultado de compresión a varios nervios craneales (V, VII, IX y $X)$ o por inflamación local crónica ocasionado por una osteítis, periostitis, y/o tendinitis $(12,13)$.

Algunos autores sugieren que la longitud aceptable del proceso estiloideo es de aproximadamente $2.5 \mathrm{~cm}$, con un límite superior dentro de parámetros normales de $3 \mathrm{~cm}(14,15)$.

Muchas herramientas de imagen pueden ser utilizadas para la valoración del síndrome de Eagle, incluyendo la radiografía panorámica con un gran valor diagnóstico, la vista radiográfica de 
cráneo Towne, la lateral de cráneo o la anteroposterior de cráneo) y la tomografía computarizada $(12,16)$.

Las indicaciones para la estiloidectomía no solo están limitadas para la sintomatología del síndrome del Eagle, si no también, debe ser considerada en un ámbito profiláctico por un posible daño carotídeo por su proximidad (17).

De acuerdo con el patrón de calcificación de Langlais et al. (18) clasifica tres tipos de apariencias radiográficas y cuatro patrones de calcificación 0 mineralización del proceso estiloideo (Figura 4).

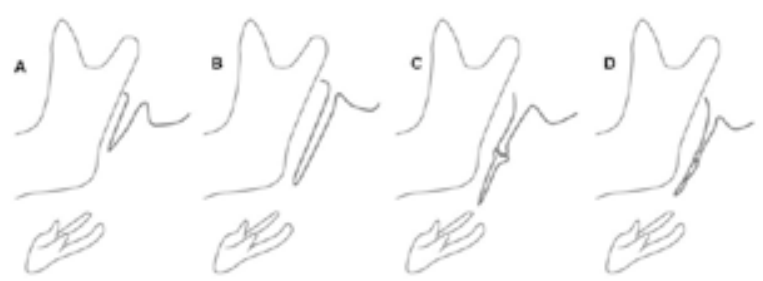

Figura 4. Representación esquemática de patrones de calcificación o mineralización del proceso estiloideo de Langlais. A. Normal; B. Elongado; C. Pseudoarticulado; D. Segmentado.

- Tipo 1: Elongado. La apariencia radiográfica de este tipo de mineralización está caracterizada por una integridad ininterrumpida del proceso estiloideo.

- Tipo 2. Pseudoarticulado. El proceso estiloideo está unido al ligamento estilohioideo o estilomandibular mineralizado por una simple pseudo articulación.

- Tipo 3. Segmentado. Consiste en una porción no continua corta o larga del proceso estiloideo 0 segmentos interrumpidos.

La estiloidectomía puede ser realizada mediante vía transcervical o trans-oral. La estiloidectomía transcervical ha sido descrita inicialmente por Loeser y Cadwell (19), desde incisio- nes muy amplias utilizadas para la disección de los nódulos linfáticos cervicales (4), abordaje para la parotidectomía, abordaje de Risdon (3), en estas técnicas, el acceso al proceso estiloideo es siempre por el espacio retro estiloideo.

Las ventajas del procedimiento transcervical es la visualización del campo quirúrgico, la adecuada exposición anatómica del proceso estiloideo y el riesgo mínimo de infección comparado con el método trans-oral. Debido a la longitud del proceso estiloideo del paciente se decidió optar por un tratamiento extraoral para facilitar su extracción, ya que se prolongaba hasta nivel del ángulo mandibular.

Dentro de las desventajas reportadas en la literatura son la cicatriz cervical, el riesgo de lesión nerviosa al nervio mandibular marginal y el nervio auricular mayor (3), el tiempo quirúrgico es de aproximadamente 30 minutos, y el daño vascular es bajo, emanando principalmente de la arterial facial, la cual puede ser fácilmente controlada a este nivel (20).

\section{CONCLUSIONES}

El abordaje transcervical para el tratamiento terapéutico de la elongación y/o mineralización del proceso estiloideo provee una mejor visualización del campo operatorio para el posible control de daños neurovasculares siguiendo con una buena técnica quirúrgica, exposición anatómica completa, y así mismo, evita la posibilidad de sufrir infecciones en comparación con el abordaje trans-oral.

\section{CONFLICTO DE INTERÉS}

Los autores declaran no tener conflicto de intereses. 


\section{DIVULGACIÓN FINANCIERA}

Autofinaciamiento.

\section{REFERENCIAS}

1. Oueslati S., Douira W., Dhieb R., Ben Farhat L., Mnif N., Rezgui L., et al. Le syndrome de Eagle. Ann d'Oto-Laryngologie Chir CervicoFaciale. 2006; 123, 3, 152-156. https://doi. org/10.1016/s0003-438x (06) 76657-9.

2. Bouguila J., Khonsari R.H., Pierrefeu A., Corre P. Eagle syndrome: A rare and atypical pain! Revue de Stomatologie et de Chirurgie Maxillo-Faciale. 2011; 112 (6): 348-352. https://doi.org/10.1016/j. stomax.2011.08.007.

3. Müderris T., Bercin S., Sevil E., Beton S., Kiris M. Surgical management of elongated styloid process: Intraoral or transcervical? Eur Arch Oto-Rhino-Laryngology. 2014; 271: 1709-1713. https://doi.org/10.1007/ s00405-013-2664-0.

4. Naik S.M., Naik S.S. Tonsillo-styloidectomy for Eagle's syndrome: A review of 15 cases in KVG Medical College Sullia. Oman Med J. 2011; 26 (2): 122-126. https://doi. org/10.5001/omj.2011.30.

5. Fusco D.J., Asteraki S., Spetzl R.F. Eagle's syndrome: Embryology, anatomy, and clinical management. Acta Neurochirurgica. 2012; 154 (7): 1119-1126. https://doi. org/10.1007/s00701-012-1385-2.

6. Badhey A., Jategaonkar A., Anglin Kovacs A.J., Kadakia S., De Deyn P.P., Ducic Y., et al. Eagle syndrome: A comprehensive review. Clinical Neurology and Neurosurgery. 2017; 159: 34-38. https://doi.org/10.1016/j. clineuro.2017.04.021.
7. Eagle W.W. Elongated Styloid Processes: Report of Two Cases. Arch Otolaryngol - Head Neck Surg. 1937; 25(5):584-587. https://doi. org/ 10.1001/archotol.1937.00650010656008.

8. Lorman J.G., Biggs J.R. The eagle syndrome. Am J Roentgenol. 1983; 140 (5): 881-882. https://doi.org/10.2214/ajr.140.5.881.

9. Kawasaki M., Hatashima S., Matsuda T. Non-surgical therapy for bilateral glossopharyngeal neuralgia caused by Eagle's syndrome, diagnosed by three-dimensional computed tomography: A case report. J Anesth. 2012; 26 (6): 918-921. https://doi. org/10.1007/s00540-012-1437-z.

10. EAGLE W.W. Symptomatic elongated styloid process; report of two cases of styloid process-carotid artery syndrome with operation. Arch Otolaryngol. 1949; 49 (5): 490-503. https://doi.org/10.1001/archotol.1949.03760110046003.

11. Eagle W.W. Elongated Styloid Process: Symptoms and Treatment. AMA Arch Otolaryngol. 1958; 67 (2): 172-176. https://doi. org/10.1001/archotol.1958.00730010178007.

12. Raina D., Gothi R., Rajan S. Eagle syndrome. Indian J Radiol Imaging. 2009; 19: 107-8. https://doi.org/10.4103/0971-3026.50826.

13. Costantinides F., Vidoni G., Bodin C., Di Lenarda R. Eagle's syndrome: signs and symptoms. Cranio : the journal of craniomandibular practice. 2013; 31 (1): 56-60. https://doi.org/10.1179/crn.2013.008.

14. Correll R.W., Jensen J.L., Taylor J.B., Rhyne RR. Mineralization of the stylohyoid-stylomandibular ligament complex. A radiographic incidence study. Oral Surgery, Oral Med Oral Pathol. 1979; 48 (4): 286-291. https:// doi.org/10.1016/0030-4220(79)90025-2.

15. Monsour P.A., Young W.G. Variability of the styloid process and stylohyoid ligament in 
panoramic radiographs. Oral Surgery, Oral Med Oral Pathol. 1986; 61 (5): 522-526. https:// doi.org/10.1016/0030-4220(86)90399-3.

16. Montalbetti L., Ferrandi D., Pergami P., Savoldi F. Elongated styloid process and Eagle's syndrome. Cephalalgia. 1995; 15 (2): 80-93. https://doi.org/10.1046/j.14682982.1995.015002080.x.

17. Mann A., Kujath S., Friedell M.L., Hardouin S., Wood C., Carter R., et al. Eagle Syndrome Presenting after Blunt Trauma. Ann Vasc Surg. 2017; Apr; 40: 295.e5-295.e8. https:// doi.org/10.1016/j.avsg.2016.07.077

18. Langlais R.P., Miles D.A., Van Dis M.L. Elongated and mineralized stylohyoid ligament complex: A proposed classification and report of a case of Eagle's syndrome. Oral Surgery, Oral Med Oral Pathol. 1986; 61 (5): 527-532. https://doi.org/10.1016/00304220(86)90400-7.

19. Loeser L.H., Cardwell E.P. Elongated Styloid Process:ACause of Glossopharyngeal Neuralgia.Arch Otolaryngol-Head Neck Surg. 1942; 36 (2): 198-202. https://doi.org/10.1001/ archotol.1942.03760020034003.

20. Pigache P., Fontaine C., Ferri J., Raoul G. Transcervical styloidectomy in Eagle's syndrome. Eur Ann Otorhinolaryngol Head Neck Dis. 2018; 135 (6): 433-436. https:// doi.org/10.1016/j.anorl.2018.05.001. 